\title{
A randomised comparison of the efficacy of a Coopdech bronchial blocker and a double-lumen endotracheal tube for minimally invasive esophagectomy
}

\author{
Tian-Hua Zhang ${ }^{1 \#}$, Xiao-Qing Liu ${ }^{2 \#}$, Long-Hui Cao ${ }^{1}$, Jian-Hua Fu ${ }^{3}$, Wen-Qian Lin ${ }^{1,4}$ \\ ${ }^{1}$ Department of Anaesthesia, Sun Yat-Sen University Cancer Center, State Key Laboratory of Oncology in South China and Collaborative \\ Innovation Center for Cancer Medicine, Guangdong Esophageal Cancer Institute, Guangzhou, China; ${ }^{2}$ Department of Anesthesia, Sun Yat-Sen \\ Memorial hospital, Sun Yat-Sen University, Guangzhou, China; ${ }^{3}$ Department of thoracic surgery, ${ }^{4}$ Department of blood transfusion, Sun Yat- \\ Sen University Cancer Center, State Key Laboratory of Oncology in South China and Collaborative Innovation Center for Cancer Medicine, \\ Guangdong Esophageal Cancer Institute, Guangzhou, China \\ Contributions: (I) Conception and design: TH Zhang, XQ Liu, WQ Lin; (II) Administrative support: TH Zhang, WQ Lin; (III) Provision of study \\ materials or patients: WQ Lin, XQ Liu; (IV) Collection and assembly of data: TH Zhang, XQ Liu; (V) Data analysis and interpretation: All authors; \\ (VI) Manuscript writing: All authors; (VII) Final approval of manuscript: All authors. \\ \#These authors contributed equally to this work. \\ Correspondence to: Wen-Qian Lin. Department of Anaesthesia, Department of Blood transfusion, Sun Yat-Sen University Cancer Center, State Key \\ Laboratory of Oncology in South China and Collaborative Innovation Center for Cancer Medicine, Guangdong Esophageal Cancer Institute, \\ Guangzhou, China. Email: Linwq@sysucc.org.cn.
}

Background: Both a bronchial blocker (BB) and a double-lumen endotracheal tube (DLT) can achieve lung collapse and one-lung ventilation (OLV) during thoracic surgery. The purpose of this study was to compare these two airway devices in terms of efficacy in video-assisted thoraco-laparoscopic esophagectomy for cancer.

Methods: A total of 55 patients underwent combined thoracoscopic and laparoscopic esophagectomy for cancer were enrolled and divided into a Coopdech bronchial blocker group (CBB group, n=27) or a DLT group (DLT group, $\mathrm{n}=28$ ). The primary outcome was the lung collapse scores at 1, 5, 10 minutes after the opening of the pleural and assessed using a verbal analogue scale via a real-time video view. Secondary outcomes including time for tube localization, incidence of tube displacement, postoperative sore throats, and surgeons' satisfaction with surgical manipulations were collected.

Results: The patients in the CBB group achieved better lung collapse scores at 5 minutes $(7.4 \pm 1.3$ vs. $6.4 \pm 0.9$ minutes, $\mathrm{P}<0.01)$ and 10 minutes $(8.9 \pm 0.8$ vs. $7.1 \pm 0.9$ minutes, $\mathrm{P}<0.01)$ after opening the pleura, and they had lower incidence of postoperative sore throats [5 (18\%) vs. $16(57 \%), \mathrm{P}<0.01$ ] when compared with patients in DLT group. However, the time for tube localization were significantly longer in CBB group than in DLT group $(210 \pm 120$ vs. $125 \pm 60 \mathrm{~s}, \mathrm{P}<0.05)$. There were no significant difference in tube displacement, hypoxemia (SpO2 <90\%) during OLV, and in surgeons' satisfaction with surgical manipulations.

Conclusions: CBB technique can be a potential alternative to the conventional DLT strategy for lung collapse and OLV during esophagectomy.

Keywords: Anaesthesia; one-lung ventilation (OLV); esophagectomy; bronchial blocker (BB); double-lumen tube (DLT)

Submitted Jan 10, 2020. Accepted for publication Jul 03, 2020.

doi: $10.21037 /$ tcr-20-378

View this article at: http://dx.doi.org/10.21037/tcr-20-378 


\section{Introduction}

Recently, minimally invasive esophagectomy (videoassisted thoraco-laparoscopic and cervical anastomosis) has increasingly been used for the treatment of esophageal cancer and has created controversy regarding which airway device is more effective and suitable for operation (1-5). Traditionally, a double-lumen endotracheal tube (DLT) has been the preferred airway device for esophageal cancer surgeries, as it can offer more rapid and better quality lung collapse. However, the influence of an inflated posterior tracheal wall due to the trachea being occupied by a bulky DLT has caused surgeons to complain about a poor surgical field view and inconvenient surgical manipulations under the thoracoscopic view, especially in difficulty to excise the left recurrent laryngeal nerve lymph node from a patient in a prone position (1-4). Moreover, the intubation of a DLT made it impossible to monitor the recurrent laryngeal nerve intraoperatively if necessary. Furthermore, DLT is related to a higher rate of postoperative sore throat and hoarseness (6). Because esophagectomy is a long operation, postoperative airway swelling may also occur after the use of a DLT and there is an added risk when exchanging a DLT with a single-lumen tube (SLT) in some patients if prolonged postoperative ventilation is necessary.

Alternatively, intubation with a SLT and utilizing a bronchial blocker (BB) to provide lung isolation and onelung ventilation $(\mathrm{OLV})$ has become increasingly popular in recent years, especially in patients with difficult DLT intubations $(7,8)$. One advantage of this technique is that it can monitor the recurrent laryngeal nerve intraoperatively if necessary by using an electromyographic endotracheal tube, while lung collapse and OLV can be achieved using a BB (9). Also, this avoids the need for tube exchange if prolonged postoperative ventilation is necessary. Previous study has compared the BB with the DLT in esophageal surgery (10). However, the efficacy of BB for lung deflation and OLV might be manufacturer/model specific as their internal BB channels have different diameters (11). In this study, we randomly compared Coopdech bronchial blocker (CBB) with the DLT strategy in terms of the efficacy during esophagectomy. We hypothesized that the CBB can be a potential alternative to the conventional DLT strategy for lung collapse and OLV during esophagectomy. We present the following article in accordance with the CONSORT reporting checklist (available at http://dx.doi.org/10.21037/ tcr-20-378).

\section{Methods}

The study was conducted in accordance with the Declaration of Helsinki (as revised in 2013). This singlecenter, randomized, double-blind study was approved by the Research Ethical Committee of the Sun Yat Sen University Cancer Center and registered at Chinese Clinical Trial Registry (ChiCTR2000032259).

Written informed consent was obtained from all patients before surgery. From May 2015 to November 2015, patients scheduled for elective esophageal cancer surgery (video-assisted thoracoscopic, laparoscopic and left neck anastomosis) were enrolled if they were more than $18 \mathrm{yr}$ of age and with an American Society of Anesthesiologists Physical Status I to II (Figure 1). Exclusion criteria included anticipated difficult intubation (Mallampatti score $\geq 3$ ), pleural adhesion, American Society of Anesthesiologists (ASA) physical status of 3-4, age $<18$ years.

After patients arrive in the operation room, standard monitoring (electrocardiogram, pulse oximeter, noninvasive blood pressure and capnography) was established. Patients' lungs were pre-oxygenated with $100 \%$ oxygen at a fresh gas flow of $4 \mathrm{~L} / \mathrm{min}$ for $3 \mathrm{~min}$ via a closely applied facemask. Anesthesia was then induced with midazolam $(0.1 \mathrm{mg} / \mathrm{kg})$, etomidate $(0.2 \mathrm{mg} / \mathrm{kg})$, sufentanil $(0.2 \mathrm{mg} / \mathrm{kg})$, and cisatracurium $(0.3 \mathrm{mg} / \mathrm{kg})$. Using computer-generated codes maintained in sequentially numbered opaque envelopes, patients were randomly allocated to CBB group and DLT group. The allocation sequence was prepared by an independent operator not involved in the study. Patients and investigators were blinded to the assigned treatment protocol. In the CBB group, the patient's trachea was first intubated with SLT (an 8.0-mm ID for males, 7.5-mm ID for females). Afterward, the CBB (the Coopdech bronchial blocker, Daiken Medical Co. Ltd, Osaka, Japan, Figure $2 A$ ) was blindly introduced into the right mainstem bronchus through inside of the SLT. In the DLT group, a left-side DLT (Mallinckrodt Medical, Athlone, Ireland; $37 \mathrm{Fr}$ for males, and $35 \mathrm{Fr}$ for females) was introduced via direct laryngoscopy. The correct position of the DLT and CBB was assessed by flexible fiberoptic bronchoscopy. Mechanical ventilation (both two lung and one lung ventilation) operated in a volume-controlled mode with a tidal volume of $6-8 \mathrm{~mL} / \mathrm{kg}$ ideal body weight at the respiratory rate of $10 / \mathrm{min}$, oxygen fraction at $0.8-1.0$ without positive end-expiratory pressure. All patients were turned in the left lateral decubitus position during thoracic 


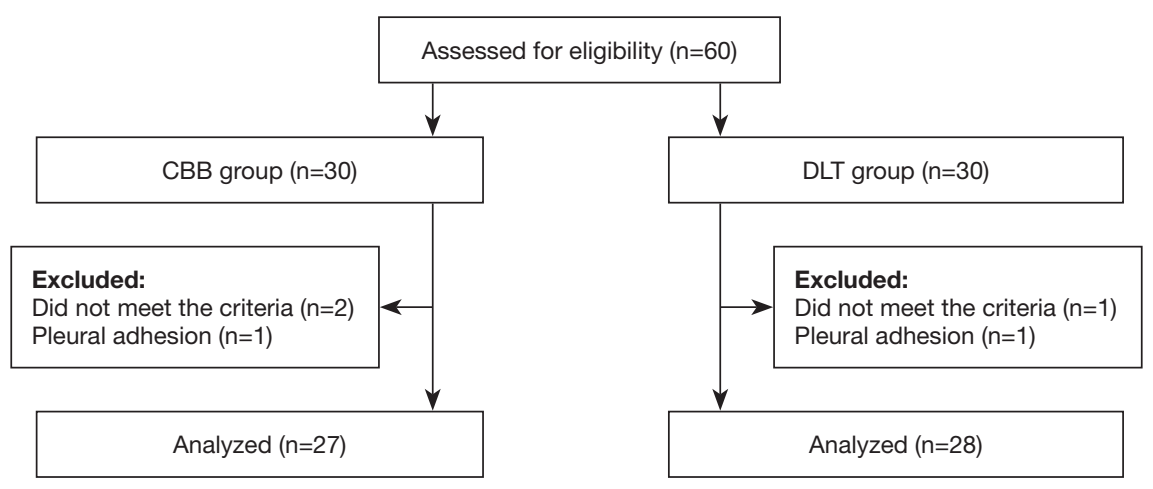

Figure 1 Flow chart of the study. CBB, Coopdech bronchial blocker; DLT, double-lumen endobronchial tube

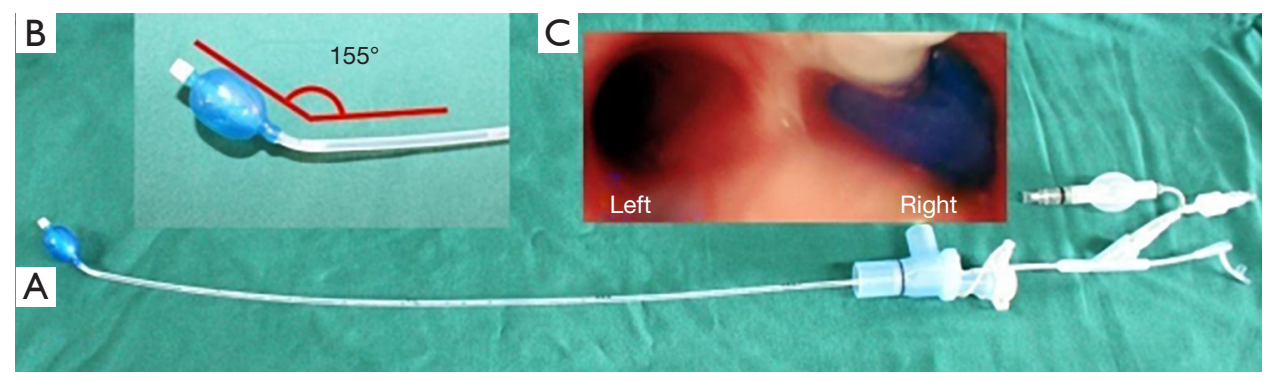

Figure 2 The Coopdech bronchial blocker (A) has a preformed angulation about 155 degree (B) and it can completely block the right main bronchus after the cuff inflated $(\mathrm{C})$.

procedure. In CBB group, OLV and lung collapse was achieved using the disconnection technique (12): at the time of the pleural incision, the ventilator was turned off and the connect to SLT was opened to air allowing both lungs to collapse for one minute (blocker cuff was deflated), then the CBB cuff was inflated with air, the central lumen was closed and the ventilator was turned on allowing only dependentlung reventilation. In DLT group, the bronchial cuff was insufflated and the appropriate Y-connected channel was clamped and opened to air.

The primary outcome in this study was the lung collapse scores at 1, 5, 10 minutes after the opening of the pleural. Lung collapse score was graded via a real-time video view and assessed using a verbal analogue scale from 0 (no lung deflation) to 10 (complete collapse) by one thoracic surgeon who was blinded to the device being used $(7,13)$. Secondary outcomes including the time for tube localization (measured from the start of the laryngoscopy until the end of the tube in correct position checked by bronchoscope), incidence of tube displacement during operation, postoperative sore throats, and surgeon's satisfaction with the surgical field view and surgical manipulation ranked as excellent (satisfied, extremely satisfied), fair (undecided, somewhat satisfied), or poor (extremely dissatisfied, dissatisfied, somewhat dissatisfied) were recorded. Secondary outcomes such as hypoxemia $\left(\mathrm{SpO}_{2}<90 \%\right.$ lasting for 5 minutes) during OLV, postoperative pulmonary complications (including pulmonary infection, hypoxemia and respiratory failure, atelectasis) were also collected.

\section{Statistical analysis}

All data were analyzed using the SPSS 19.0 version (SPSS, Inc., Chicago, IL). Continuous data are presented as mean \pm standard deviation (SD) or median [range], and the categorical data are presented as number (percentage). The primary outcome (lung collapse scores) was analyzed using independent $t$ test. The data of time for tube localization were analyzed using independent $t$ test. The data of tube displacement, hypoxemia during OLV and the categorical postoperative complication data were analyzed using Fisher's exact test. Two-sided $\mathrm{P}$ values $<0.05$ were considered to indicate statistical significance.

A previous study has showed a $30 \%$ of total lung collapse 
Table 1 Patients and surgical characteristics

\begin{tabular}{lccc}
\hline Characteristics & $\begin{array}{c}\text { CBB group } \\
(\mathrm{n}=27)\end{array}$ & $\begin{array}{c}\text { DLT group } \\
(\mathrm{n}=28)\end{array}$ & P value \\
\hline Age (years) & $61.6 \pm 8.1$ & $62.3 \pm 8.2$ & 0.17 \\
Sex (male/female) & $19 / 8$ & $20 / 8$ & 0.60 \\
Weigh (kg) & $61.2 \pm 5.1$ & $62.3 \pm 6.2$ & 0.74 \\
Height (cm) & $165 \pm 5$ & $167 \pm 7$ & 0.66 \\
Concomitant disease, $\mathrm{n}(\%)$ & & & \\
Diabetes & $7(25.9)$ & $6(21.4)$ & 0.31 \\
Hypertension & $10(37)$ & $12(42.8)$ & 0.24 \\
Smoking & $15(55.5)$ & $13(46.4)$ & 0.47 \\
Neoadjuvant treatment & $10(37)$ & $11(39)$ & 0.27 \\
Surgery, min & & & \\
Anesthesia time & $280 \pm 85$ & $298 \pm 76$ & 0.38 \\
Surgical time & $240 \pm 75$ & $251 \pm 70$ & 0.34 \\
OLV & $85 \pm 37$ & $91 \pm 47$ & 0.46 \\
\hline
\end{tabular}

Data are presented as mean (standard deviation) or number (proportion). CBB, Coopdech bronchial blocker. DLT, double lumen endotracheal tube, OLV, one lung ventilation.

at $10 \mathrm{~min}$ in the DLT group (7). Assuming a $40 \%$ difference between groups with an alpha of 0.05 and a power of 0.80 , we calculated that 27 patients in each group are required. On accounting for a dropout rate of $10 \%, 60$ patients were recruited in total.

\section{Results}

A total of 60 patients were assessed for eligibility. Three patients did not meet the criteria and 2 were excluded because of pleural adhesion. The remaining 55 subjects were randomly divided into two groups (Figure 1). The baseline characteristics were similar among two groups (Table 1).

The patients in the CBB group achieved better lung collapse scores for OLV at five minutes $(7.4 \pm 1.3$ vs. $6.4 \pm 0.9$, $\mathrm{P}<0.01)$ and ten minutes $(8.9 \pm 0.8$ vs. $7.2 \pm 0.9, \mathrm{P}<0.01)$ after opening the pleura, and they had lower incidence of postoperative sore throats [5 (18\%) vs. $16(57 \%), \mathrm{P}<0.01$ ] when compared with patients in DLT group (Table 2). However, the time for tube localization was significantly longer in CBB group than DLT group $(210 \pm 120$ vs. $125 \pm 60 \mathrm{~s}, \mathrm{P}<0.05)$. There were no significant differences in tube displacement, hypoxemia $\left(\mathrm{SpO}_{2}<90 \%\right)$ during the
Table 2 Efficacy of the airway device and postoperative complications

\begin{tabular}{lccc}
\hline Characteristics & $\begin{array}{c}\text { CBB group } \\
(\mathrm{n}=27)\end{array}$ & $\begin{array}{c}\mathrm{nLT} \text { group } \\
(\mathrm{n}=28)\end{array}$ & P value \\
\hline Lung collapse score & & & \\
$1 \mathrm{~min}$ & $5.7 \pm 1.3$ & $5.5 \pm 1.1$ & 0.13 \\
$5 \mathrm{~min}$ & $7.4 \pm 1.3$ & $6.4 \pm 0.9$ & 0.002 \\
$10 \mathrm{~min}$ & $8.9 \pm 0.8$ & $7.1 \pm 0.9$ & 0.001 \\
Time for tube localization (s) & $210 \pm 120$ & $125 \pm 60$ & 0.02 \\
Surgeon's satisfaction, $\mathrm{n}(\%)$ & & & 0.13 \\
Poor & $2(7.4)$ & $4(14.2)$ & \\
Fair & $8(29.6)$ & $9(32.1)$ & \\
Excellent & $17(63)$ & $15(53.6)$ & \\
SpO & & \\
Displace of tube & $5(18.5)$ & $7(24.1)$ & 0.21 \\
Sore throat & $4(14.8)$ & $6(20.6)$ & 0.13 \\
Pulmonary complications & $6(22.2)$ & $8(28.5)$ & 0.24 \\
Tore of lumen & $2(7.4)$ & $0(0.0)$ & \\
\hline
\end{tabular}

Data are presented as mean (standard deviation) or number (proportion). CBB, Coopdech bronchial blocker. DLT, double lumen endotracheal tube, OLV, one lung ventilation.

OLV, lung collapse score at 1 min after opening the pleura, postoperative pulmonary complications, and in surgeons' satisfaction with surgical manipulations between two groups (Table 2).

The Coopdech cuff rupture occurred in two patients. Three patients in the CBB group and 6 patients in the DLT group required prolonged postoperative ventilation and replacement of the DLT with an SLT.

\section{Discussion}

Our results demonstrate that the use of CBB could achieve better quality of lung collapse scores for OLV at 5 and 10 min after the opening of the pleural as compared with that of DLT. Moreover, the patients in the CBB group had lower incidence of postoperative sore throats. Although the use of a CBB is associated with a longer time for tube localization when compared with DLT, but it is faster than other model of BB to localization. Our results are similar to those of other studies (6-10).

One novel aspect of our study was the use of an approximately one- minute disconnection technique when 
using the $\mathrm{CBB}$ for lung isolation. Prior studies have reported one period of apnea (prior to initiating OLV) and one study used two periods of apnea (30 s prior to initiating OLV and at the time of pleural incision) when using the blocker for lung isolation (7,9-11). These studies demonstrated similar or inferior results to DLT. In a recent study, a twominute disconnection technique was used in DLT and was able to speed the lung collapse (14). Our results from the use of the one-minute disconnection technique revealed an important increase in the quality of the lung deflation. We believe that there are several possible explanations for the improved lung collapse observed with the CBB. First, the cuff of bronchial blocker can block the right mainstem bronchus more closer than a DLT, this make the lung collapse better. Second, the air movement from the mediastinal shifts during OLV with the non-dependent lung side opened to room air when a DLT was used, which may influence lung collapse. Third, compared with the closed bronchial blocker, there are significant quantities of $\mathrm{O}_{2}$ in the lung that are replaced duo to the passive inflow of ambient air into the non-dependent lung, which therefore impedes the phase 2 lung deflation with the DLT. Although the speed and quality of lung deflation depends on the lung condition, one study demonstrates that the use of $50 \% \mathrm{~N} 2 \mathrm{O}$ before OLV facilitates lung collapse when a bronchial blocker is used (15). Whether suction is effective in lung deflation need more evidence when using a blocker (16).

It is important to consider safety as well as the efficacy when comparing devices used for lung isolation. In this study, we found that fewer patients in the CBB developed postoperative sore throats. These results suggest that intubation with a DLT could cause more minor airway injuries. During esophagectomy, many patients need monitor the recurrent laryngeal nerve intraoperatively to avoid nerve injury. This can be achieved by using an electromyographic endotracheal tube, while lung collapse and OLV can be achieved using a CBB. However, it is impossible to monitor the recurrent laryngeal nerve when a DLT is used (9). There were no significant difference in tube displacement, hypoxemia $\left(\mathrm{SpO}_{2}<90 \%\right)$ during $\mathrm{OLV}$, and in surgeons' satisfaction with surgical manipulations between two groups, suggesting that both airway devices are in same efficacy in those aspects. We believe that there are several possible explanations for those: the distal tip of a CBB has a preformed angulation (about 155 degree, Figure $2 B$ ) that is designed to facilitate its easy insertion and positioning in the desired main bronchus, with fewer dislocations during repositioning the patient and surgical manipulations (14). Moreover, the repositioning of a displaced Coopdech is expected to be easier. Furthermore, a single size CBB will fit almost all adult patients, and its large-volume, lowpressure cuff can make it complete block the right main bronchus after inflated (Figure 2C). As an esophageal surgery, tube displacement happened very often at both groups because of surgical manipulations, however, there was no difference between two groups. In this study, we did not compare postoperative hoarseness, as many factors can cause hoarseness. There were 6 patients in the DLT group and 3 patients in the CBB group who required prolonged postoperative ventilation. Intubation with an SLT was more convenient than a DLT, which should be exchanged for an SLT, because of the cuff was not deflated when reposition in the CBB, which cause the cuff rupture in 2 Coopdech blocker patients. There is a report of an accidental fracture of the tip of a CBB during insertion (17).

One limitation of the current study was the method of assessing outcomes such as lung collapse scores by using the surgeons' rating scale, which was not completely objective. Secondly, our study involved a single centre-based design which may have led to an uncertain amount of selection bias. Other limitation is that we did not compare the cost of two airway devices, as a BB may cost much than a DLT, which should be considered when chose a device.

\section{Conclusions}

In conclusion, the results of this study suggested that despite requiring a longer period to achieve tube localization, the use of a CBB is safe and an effective device, and can be a potential alternative to the conventional DLT strategy for lung collapse and OLV during esophagectomy.

\section{Acknowledgments}

Funding: This work was funded by Guangdong Esophageal Cancer Institute Science and Technology Program (M 201603).

\section{Footnote}

Reporting Checklist: The authors have completed the CONSORT reporting checklist. Available at http://dx.doi. org/10.21037/tcr-20-378

Data Sharing Statement: Available at http://dx.doi. 
org/10.21037/tcr-20-378

Peer Review File: Available at http://dx.doi.org/10.21037/tcr20-378

Conflicts of Interest: All authors have completed the ICMJE uniform disclosure form (available at http://dx.doi. org/10.21037/tcr-20-378). The authors have no conflicts of interest to declare.

Ethical Statement: The authors are accountable for all aspects of the work in ensuring that questions related to the accuracy or integrity of any part of the work are appropriately investigated and resolved. The study was conducted in accordance with the Declaration of Helsinki (as revised in 2013). This single-center, randomized, double-blind study was approved by the Research Ethical Committee of the Sun Yat Sen University Cancer Center and registered at Chinese Clinical Trial Registry (ChiCTR2000032259). Written informed consent was obtained from all patients before surgery.

Open Access Statement: This is an Open Access article distributed in accordance with the Creative Commons Attribution-NonCommercial-NoDerivs 4.0 International License (CC BY-NC-ND 4.0), which permits the noncommercial replication and distribution of the article with the strict proviso that no changes or edits are made and the original work is properly cited (including links to both the formal publication through the relevant DOI and the license). See: https://creativecommons.org/licenses/by-nc-nd/4.0/.

\section{References}

1. Cai L, Li Y, Sun L, et al. Better perioperative outcomes in thoracoscopic-esophagectomy with two-lung ventilation in semi-prone position. J Thorac Dis 2017;9:117-22.

2. Singh M, Uppal R, Chaudhary K, et al. Use of single-lumen tube for minimally invasive and hybrid esophagectomies with prone thoracoscopic dissection: case series. J Clin Anesth 2016;33:450-55.

3. Zhang R, Liu S, Sun H, et al. The application of singlelumen endotracheal tube anaesthesia with artificial pneumothorax in thoracolaparoscopic oesophagectomy. Interac Interact Cardiovasc Thorac Surg 2014;19:308-10.

4. Saikawa D, Okushiba S, Kawata M, et al. Efficacy and safety of artificial pneumothorax under two-lung ventilation in thoracoscopic esophagectomy for esophageal cancer in the prone position. Gen Thorac Cardiovasc Surg 2014;62:163-70.

5. Takeuchi H, Kitagawa Y. Two-lung ventilation in the prone position: is it the standard anesthetic management for thoracoscopic esophagectomy? Gen Thorac Cardiovasc Surg 2014;62:133-4.

6. Knoll H, Ziegeler S, Schreiber JU, et al. Airway injuries after one-lung ventilation: a comparison between double-lumen tube and endobronchial blocker: a randomized, prospective, controlled trial. Anesthesiology 2006; 105:471-7.

7. Bussières JS, Somma J, Del Castillo JL, et al. Bronchial blocker versus left double-lumen endotracheal tube in video-assisted thoracoscopic surgery: a randomizedcontrolled trial examining time and quality of lung deflation. Can J Anaesth 2016;63:818-27.

8. Clayton-Smith A, Bennett K, Alston RP, et al. A comparison of the efficacy and adverse effects of doublelumen endobronchial tubes and bronchial blockers in thoracic surgery: a systematic review and meta-analysis of randomized controlled trials. J Cardiothorac Vasc Anesth 2015;29:955-66.

9. Moritz A, Schmidt J, Schreiner, W, et al. Combined recurrent laryngeal nerve monitoring and one-lung ventilation using the EZ-Blocker and an electromyographic endotracheal tube. J Cardiothorac Surg 2019;14:111.

10. Lu Y, Dai W, Zong Z, et al. Bronchial Blocker Versus Left Double-Lumen Endotracheal Tube for One-Lung Ventilation in Right Video-Assisted Thoracoscopic Surgery. J Cardiothorac Vasc Anesth 2018;32:297-301.

11. Narayanaswamy M, McRae K, Slinger P, et al. Choosing a lung isolation device for thoracic surgery: a randomized trial of three bronchial blockers versus double-lumen tubes. Anesth Analg 2009;108:1097-101.

12. Yoo JY, Kim DH, Choi H, et al. Disconnection technique with a bronchial blocker for improving lung deflation: a comparison with a double-lumen tube and bronchial blocker without disconnection. J Cardiothorac Vasc Anesth 2014;28:904-7.

13. Campos JH, Hallam EA, Ueda K. Lung isolation in the morbidly obese patient: a comparison of a left-sided double-lumen tracheal tube with the Arndt ${ }^{\circledR}$ wire-guided blocker. Br J Anaesth 2012;109:630-5.

14. Li Q, Zhang X, Wu J, et al. Two-minute disconnection technique with a double-lumen tube to speed the collapse of the non-ventilated lung for one-lung ventilation in thoracoscopic surgery. BMC Anesthesiol 2017;17:80.

15. Yoshimura T, Ueda K, Kakinuma A, et al. Bronchial 
blocker lung collapse technique: nitrous oxide for facilitating lung collapse during one-lung ventilation with a bronchial blocker. Anesth Analg 2014;118:666-70.

16. Quan X, Yi J, Huang Y, et al. Bronchial suction does not facilitate lung collapse when using a double-lumen tube during video-assisted thoracoscopic surgery: a randomized controlled trial. J Thorac Dis 2017;9:5244-8.

17. Venkataraju A, Rozario C, Saravanan P. Accidental fracture of the tip of the Coopdech bronchial blocker during insertion for one lung ventilation. Can J Anaesth 2010;57:350-4.
Cite this article as: Zhang TH, Liu XQ, Cao LH, Fu JH, Lin WQ. A randomised comparison of the efficacy of a Coopdech bronchial blocker and a double-lumen endotracheal tube for minimally invasive esophagectomy. Transl Cancer Res 2020;9(8):4686-4692. doi: 10.21037/tcr-20-378 